\title{
Ergodic averages with prime divisor weights in $L^{1}$
}

\author{
Zoltán Buczolich*, Department of Analysis, Eötvös Loránd \\ University, Pázmány Péter Sétány 1/c, 1117 Budapest, Hungary \\ email: buczo@cs.elte.hu \\ www.cs.elte.hu/ buczo
}

April 18, 2018

\begin{abstract}
We show that $\omega(n)$ and $\Omega(n)$, the number of distinct prime factors of $n$ and the number of distinct prime factors of $n$ counted according to multiplicity are good weighting functions for the pointwise ergodic theorem in $L^{1}$. That is, if $g$ denotes one of these functions and $S_{g, K}=$ $\sum_{n \leq K} g(n)$ then for every ergodic dynamical system $(X, \mathcal{A}, \mu, \tau)$ and every $f \in L^{1}(X)$

$$
\lim _{K \rightarrow \infty} \frac{1}{S_{g, K}} \sum_{n=1}^{K} g(n) f\left(\tau^{n} x\right)=\int_{X} f d \mu \text { for } \mu \text { a.e. } x \in X .
$$

This answers a question raised by C. Cuny and M. Weber who showed this result for $L^{p}, p>1$.

\footnotetext{
*Research supported by National Research, Development and Innovation OfficeNKFIH, Grant 104178.

Mathematics Subject Classification: Primary : 37A30; Secondary : 11A25, 28D05, $37 \mathrm{~A} 05$.

Keywords: Ergodic averages, pointwise convergence, maximal inequality, number of distinct prime factors.
} 


\section{Introduction}

In [1] C. Cuny and M. Weber investigated whether some arthimetic weights are good weights for the pointwise ergodic theorem in $L^{p}$. In this paper we show that the prime divisor functions $\omega$ and $\Omega$ are both good weights for the $L^{1}$ pointwise ergodic theorem. The same fact for the spaces $L^{p}, p>1$ was proved in [1] and our paper answers a question raised in that paper. Recall that if $n=p_{1}^{\alpha_{1}} \cdots p_{k}^{\alpha_{k}}$ then $\omega(n)=k$ and $\Omega(n)=\alpha_{1}+\ldots+\alpha_{k}$. We denote by $g$ one of these functions. Given $K$ we put

$$
S_{g, K}=\sum_{n \leq K} g(n) .
$$

We suppose that $(X, \mathcal{A}, \mu)$ is a measure space and $\tau: X \rightarrow X$ is a measure preserving ergodic transformation. Given $f \in L^{1}(X)$ we consider the $g$ weighted ergodic averages

$$
\mathcal{M}_{g, K} f(x)=\frac{1}{S_{g, K}} \sum_{n=1}^{K} g(n) f\left(\tau^{n} x\right) .
$$

We show that for $g=\omega$, or $\Omega$ these averages $\mu$ a.e. converge to $\int_{X} f d \mu$, that is $g$ is a good universal weight for the pointwise ergodic theorem in $L^{1}$. See Theorem 6 .

For some similar ergodic theorems with other weights like the Möbius function, or its absolute value, or the Liouville function we refer to the papers

of El Abdalaoui, Kułaga-Przymus, Lemańczyk and de la Rue, [3], and of Rosenblatt and Wierdl [8].

\section{Preliminary results}

We recall Theorem 430 from p. 72 of [5]

$$
\begin{gathered}
\sum_{n \leq K} \omega(n)=K \log \log K+B_{1} K+o(K) \text { and } \\
\sum_{n \leq K} \Omega(n)=K \log \log K+B_{2} K+o(K) .
\end{gathered}
$$


Hence, for both cases we can assume that there exists a constant $B$ (which depends on whether $g=\omega$, or $g=\Omega$ ) such that

$$
\sum_{n \leq K} g(n)=K \log \log K\left(1+\frac{B}{\log \log K}+\frac{o(K)}{K \log \log K}\right) .
$$

From this it follows that there exists $C_{g}>0$ such that for all $K \in \mathbb{N}$

$$
\left(\sum_{n \leq K} g(n)\right)^{\lfloor\log \log K\rfloor}=\left(S_{g, K}\right)^{\lfloor\log \log K\rfloor}>C_{g}(K\lfloor\log \log K\rfloor)^{\lfloor\log \log K\rfloor} .
$$

We need some information about the distribution of the functions $\omega$ and $\Omega$. We use (3.9) from p. 689 of [6] by K. K. Norton which is based on a result of Halász [4] which is cited as (3.8) Lemma in [6]. Next we state (3.9) from [6] with $\delta=0.1$ and $z=2-\delta=1.9$.

Proposition 1. There exists a constant $\widetilde{C}_{H}$ such that for every $K \geq 1$

$$
\sum_{n \leq K} 1.9^{\omega(n)} \leq \sum_{n \leq K} 1.9^{\Omega(n)} \leq \widetilde{C}_{H} K \exp (0.9 \cdot E(K)), \text { where }
$$

$E(K)=\sum_{p \leq K} \frac{1}{p}$

Recall that by Theorem 427 in [5]

$$
E(K)=\sum_{p \leq K} \frac{1}{p}=\log \log K+B_{1}+o(1) .
$$

The constant $B_{1}$ is the same which appears in (21). The way we will use this is the following: there exists a constant $C_{P}$ such that for $K>3$

$$
E(K)=\sum_{p \leq K} \frac{1}{p}<C_{P} \log \log K .
$$

Combining this with ([6) we obtain that for $g=\omega$, or $\Omega$ we have for $K>3$

$$
\sum_{n \leq K} 1.9^{g(n)}<\widetilde{C}_{H} \cdot K \cdot \exp \left(0.9 \cdot C_{P} \log \log K\right) \leq C_{H} \cdot K \exp \left(0.9 \cdot C_{P}\lfloor\log \log K\rfloor\right),
$$

with a suitable constant $C_{H}$ not depending on $K$.

In [1] a result of Delange [2] was used to deduce Theorem 2.7 in [1]. The result of Delange is the following 
Theorem 2. For every $m \geq 1$ we have

$$
\sum_{n \leq K} g(n)^{m}=K(\log \log K)^{m}+O\left(K(\log \log K)^{m-1}\right) .
$$

We were unable to use this result since the constant in $O\left(K(\log \log K)^{m-1}\right)$ cannot be chosen not depending on $m \geq 1$.

Hence we use (9) in the proof of the following lemma.

Lemma 3. There exists a constant $C_{\Omega, \max }$ such that for all $K \geq 16$

$$
\sum_{n \leq K} \omega(n)^{\lfloor\log \log K\rfloor} \leq \sum_{n \leq K} \Omega(n)^{\lfloor\log \log K\rfloor}<K\left(C_{\Omega, \max }\lfloor\log \log K\rfloor\right)^{\lfloor\log \log K\rfloor}
$$

We remark that the assumption $K \geq 16$ implies that $\log \log K>1.01>$ 1.

Proof. Since $\omega(n) \leq \Omega(n)$ the first inequality is obvious in (10),

We assume that $K \geq 16$ is fixed and for ease of notation we put $\nu=$ $\lfloor\log \log K\rfloor$. Set

$$
N_{l, K}=\left\{n \leq K: 2^{l} \nu \leq \Omega(n)<2^{l+1} \nu\right\} .
$$

By (9) $N_{l, K} \cdot 1.9^{2^{l} \nu}<C_{H} K \exp \left(0.9 \cdot C_{P} \nu\right)$. This implies that

$$
N_{l, K}<C_{H} K \cdot \exp \left(\left(0.9 \cdot C_{P}-2^{l} \log 1.9\right) \nu\right) .
$$

Since $\log 1.9>0.6$ we can choose $l_{0}$ such that for $l \geq l_{0}$

$$
0.9 \cdot C_{P}-2^{l} \log 1.9+(l+1) \log 2<-0.5 \cdot 2^{l}=-2^{l-1} .
$$

From (12) and (13) we infer

$$
\begin{gathered}
\sum_{n \leq K} \Omega(n)^{\nu}<K \cdot(2 \nu)^{\nu}+\sum_{l=1}^{\infty} N_{l, K}\left(2^{l+1} \nu\right)^{\nu} \leq \\
K \cdot(2 \nu)^{\nu}+\sum_{l=1}^{l_{0}-1} K\left(2^{l+1} \nu\right)^{\nu}+\sum_{l=l_{0}}^{\infty} C_{H} K \nu^{\nu} \exp \left(\left(\left(\log 2^{l+1}\right)+0.9 C_{P}-2^{l} \log 1.9\right) \nu\right)<
\end{gathered}
$$


(using (13) with a suitable constant $C_{\Omega, 1}>2$ we obtain)

$$
C_{\Omega, 1}^{\nu} K \nu^{\nu}+\sum_{l=l_{0}}^{\infty} C_{H} K \nu^{\nu} \exp \left(-2^{l-1} \nu\right)<
$$

(recalling that $\nu=\lfloor\log \log K\rfloor \geq\lfloor\log \log 16\rfloor=1$, with a suitable constant $C_{\Omega, \max }$ we have)

$$
\begin{gathered}
K \nu^{\nu}\left(C_{\Omega, 1}^{\nu}+C_{H} \sum_{l=l_{0}}^{\infty} \exp \left(-2^{l-1}\right)\right)<C_{\Omega, \max }^{\nu} K \nu^{\nu}= \\
K\left(C_{\Omega, \max }\lfloor\log \log K\rfloor\right)^{\lfloor\log \log K\rfloor} .
\end{gathered}
$$

We need the following (probably well-known) elementary inequality to which we could not find a reference and hence provided the short proof.

Lemma 4. Suppose $K, \nu \in \mathbb{N}, b_{1}, \ldots, b_{K}$ are nonnegative numbers and we have permutations $\pi_{j}:\{1, \ldots, K\} \rightarrow\{1, \ldots, K\}, j=1, \ldots, \nu$. Then

$$
b_{\pi_{1}(1)} \cdots b_{\pi_{\nu}(1)}+\ldots+b_{\pi_{1}(K)} \cdots b_{\pi_{\nu}(K)} \leq b_{1}^{\nu}+\ldots+b_{K}^{\nu} .
$$

Proof. Without limiting generality we can suppose that $0 \leq b_{1} \leq \ldots \leq b_{K}$. First observe that if $A>B \geq 0$ and $C>D \geq 0$ then

$$
\text { from }(A-B)(C-D) \geq 0 \text { it follows that } A C+B D \geq A D+B C \text {. }
$$

Set $\pi_{j, 1}(k)=\pi_{j}(k)$ for $j=1, \ldots, \nu$ and $k=1, \ldots, K$. If $\pi_{j, l}$ is defined for an $l \in \mathbb{N}$ then set

$$
\mathfrak{M}_{l}^{*}=\max _{k} b_{\pi_{1, l}(k)} \cdots b_{\pi_{\nu, l}(k)} .
$$

We want to define a sequence of permutations such that for every $l$

$$
\begin{gathered}
b_{\pi_{1, l-1}(1)} \cdots b_{\pi_{\nu, l-1}(1)}+\ldots+b_{\pi_{1, l-1}(K)} \cdots b_{\pi_{\nu, l-1}(K)} \leq \\
b_{\pi_{1, l}(1)} \cdots b_{\pi_{\nu, l}(1)}+\ldots+b_{\pi_{1, l}(K)} \cdots b_{\pi_{\nu, l}(K)} .
\end{gathered}
$$

Suppose that $\mathfrak{M}_{l}^{*}<b_{K}^{\nu}$. Select $k^{*}$ such that $\mathfrak{M}_{l}^{*}=b_{\pi_{1, l}\left(k^{*}\right)} \cdots b_{\pi_{\nu, l}\left(k^{*}\right)}$. Then we can select $j^{*}$ such that $b_{\pi_{j^{*}, l}\left(k^{*}\right)}<b_{K}$ and $k^{* *}$ such that $b_{\pi_{j^{*}, l}\left(k^{* *}\right)}=b_{K}$. Set $A=b_{K}=b_{\pi_{j^{*}, l}\left(k^{* *}\right)}, B=b_{\pi_{j^{*}, l}\left(k^{*}\right)}, C=b_{\pi_{1, l}\left(k^{*}\right)} \cdots b_{\pi_{\nu, l}\left(k^{*}\right)} / B=\mathfrak{M}_{l}^{*} / B$ 
and $D=b_{\pi_{1, l}\left(k^{* *}\right)} \cdots b_{\pi_{\nu, l}\left(k^{* *}\right)} / A$. Then $A>B \geq 0$ and $C>D \geq 0$. Set $\pi_{j^{*}, l+1}\left(k^{* *}\right)=\pi_{j^{*}, l}\left(k^{*}\right), \pi_{j^{*}, l+1}\left(k^{*}\right)=\pi_{j^{*}, l}\left(k^{* *}\right)$, and for any other $j$ and $k$ set $\pi_{j, l+1}(k)=\pi_{j, l}(k)$. From (16) it follows that (17) holds with $l$ replaced by $l+1$ and $\mathfrak{M}_{l+1}^{*}>\mathfrak{M}_{l}^{*}$. Hence in finitely many steps there is $l_{1}$ such that $\mathfrak{M}_{l_{1}}^{*}=b_{K}^{\nu}$.

After step $l_{1}$ arguing as above we can still define the permutations $\pi_{j, l}$ so that (17) holds at each step and can reach a step $l_{2}$ such that $\mathfrak{M}_{l_{2}}^{*}=b_{K}^{\nu}$ and the second largest term among $b_{\pi_{1, l_{2}}(k)} \cdots b_{\pi_{\nu, l_{2}}(k)}, k=1, \ldots, K$ equals $b_{K-1}^{\nu}$. Repeating this procedure one can obtain (15).

We will use the transference principle and hence we need to consider functions on the integers. Suppose $\varphi: \mathbb{Z} \rightarrow[0,+\infty)$ is a function on the integers with compact/bounded support. Again $g$ will denote $\omega$, or $\Omega$. Put

$$
M_{g, K} \varphi(j)=\frac{1}{S_{g, K}} \sum_{n=1}^{K} g(n) \varphi(j+n) \text { for } j \in \mathbb{Z}
$$

First we prove a "localized" maximal inequality.

Lemma 5. There exists a constant $C_{g, \max }>0$ such that for any $\varphi: \mathbb{Z} \rightarrow$ $[0,+\infty), K \geq 16$ and $k \in \mathbb{Z}$

$$
\sum_{j=1}^{K}\left(M_{g, K} \varphi(k+j)\right)^{\lfloor\log \log K\rfloor} \leq\left(\sum_{j=2}^{2 K} \varphi(k+j)\right)\left(\frac{C_{g, \max }}{K} \sum_{j=2}^{2 K} \varphi(k+j)\right)^{\lfloor\log \log K\rfloor-1} .
$$

Proof. Without limiting generality we can suppose that $k=0$ and $K \geq 16$ is fixed. We use again the notation $\nu=\nu_{K}=\lfloor\log \log K\rfloor$. We put

$$
\widetilde{g}(n)=\widetilde{g}_{K}(n)=\left\{\begin{array}{cl}
g(n) & \text { if } 1 \leq n \leq K \\
0 & \text { otherwise }
\end{array}\right.
$$

We need to estimate

$$
\begin{gathered}
\sum_{j=1}^{K}\left(\frac{1}{S_{g, K}} \sum_{n=1}^{K} g(n) \varphi(j+n)\right)^{\nu}= \\
\frac{1}{S_{g, K}^{\nu}} \sum_{j=1}^{K} \sum_{n_{1}=1}^{K} \cdots \sum_{n_{\nu}=1}^{K} g\left(n_{1}\right) \cdots g\left(n_{\nu}\right) \cdot \varphi\left(j+n_{1}\right) \cdots \varphi\left(j+n_{\nu}\right)=
\end{gathered}
$$




$$
\begin{aligned}
& \frac{1}{S_{g, K}^{\nu}} \sum_{n^{\prime}=1}^{K} \sum_{j_{1}=2}^{2 K} \ldots \sum_{j_{\nu}=2}^{2 K} \varphi\left(j_{1}\right) \cdots \varphi\left(j_{\nu}\right) \cdot \widetilde{g}\left(n^{\prime}\right) \widetilde{g}\left(n^{\prime}+j_{2}-j_{1}\right) \cdots \widetilde{g}\left(n^{\prime}+j_{\nu}-j_{1}\right)= \\
& \frac{1}{S_{g, K}^{\nu}} \sum_{j_{1}=2}^{2 K} \ldots \sum_{j_{\nu}=2}^{2 K} \varphi\left(j_{1}\right) \cdots \varphi\left(j_{\nu}\right) \cdot \sum_{n^{\prime}=1}^{K} \widetilde{g}\left(n^{\prime}\right) \widetilde{g}\left(n^{\prime}+j_{2}-j_{1}\right) \cdots \widetilde{g}\left(n^{\prime}+j_{\nu}-j_{1}\right) \leq
\end{aligned}
$$

(using Lemma 4 and (19) )

$$
\begin{gathered}
\frac{1}{S_{g, K}^{\nu}} \sum_{j_{1}=2}^{2 K} \ldots \sum_{j_{\nu}=2}^{2 K} \varphi\left(j_{1}\right) \cdots \varphi\left(j_{\nu}\right) \cdot \sum_{n^{\prime}=-K+2}^{2 K-1}\left(\widetilde{g}\left(n^{\prime}\right)\right)^{\nu}= \\
\frac{1}{S_{g, K}^{\nu}} \sum_{j_{1}=2}^{2 K} \cdots \sum_{j_{\nu}=2}^{2 K} \varphi\left(j_{1}\right) \cdots \varphi\left(j_{\nu}\right) \cdot \sum_{n^{\prime}=1}^{K}\left(g\left(n^{\prime}\right)\right)^{\nu} \leq
\end{gathered}
$$

(by using Lemma 3])

$$
K \cdot C_{\Omega, \max }^{\nu} \nu^{\nu} \frac{1}{S_{g, K}^{\nu}}\left(\sum_{j=2}^{2 K} \varphi(j)\right)^{\nu}<
$$

(by (5))

$$
K \cdot C_{\Omega, \max }^{\nu} \nu^{\nu} \frac{1}{C_{g}(K \nu)^{\nu}}\left(\sum_{j=2}^{2 K} \varphi(j)\right)^{\nu}<
$$

(with a suitable constant $C_{g, \max }>0$ )

$$
<\left(\sum_{j=2}^{2 K} \varphi(j)\right) \cdot\left(C_{g, \max } \frac{1}{K} \sum_{j=2}^{2 K} \varphi(j)\right)^{\nu-1} .
$$

\section{Main result}

Theorem 6. For every ergodic dynamical system $(X, \mathcal{A}, \mu, \tau)$ and every $f \in$ $L^{1}(X)$

$$
\lim _{K \rightarrow \infty} \mathcal{M}_{g, K} f(x)=\int_{X} f d \mu \text { for } \mu \text { a.e. } x \in X \text {. }
$$


Proof. By Theorem 2.5 and Remark 2.6 of [1] we know that $\omega$ and $\Omega$ are good weights for the pointwise ergodic theorem in $L^{p}$ for $p>1$. This means that we have a dense set of functions in $L^{1}$ for which the pointwise ergodic theorem holds. In Theorem 2.5 of [1] it is not stated explicitely that the limit function of the averages $\mathcal{M}_{g, K} f$ is $\int_{X} f d \mu$, but from the proof of this theorem it is clear that $\mathcal{M}_{g, K} f$ not only converges a.e., but its limit is indeed $\int_{X} f d \mu$ (at least for $f \in L^{\infty}(\mu)$ ). Indeed, from (2.2) in [1] it follows that $\mathcal{M}_{g, K} f$ can be written as the sum of an ordinary Birkhoff-average of $f$ and an error term which tends to zero as $K \rightarrow \infty$.

Hence by standard application of Banach's principle (see for example [7] p. 91) the following weak $L^{1}$-maximal inequality proves Theorem 6 ,

Proposition 7. There exists a constant $C_{\max }$ such that for every ergodic dynamical system $(X, \mathcal{A}, \mu, \tau)$ for every $f \in L^{1}(\mu)$ and $\lambda \geq 0$

$$
\mu\left\{x: \sup _{K \geq 1} \mathcal{M}_{g, K} f(x)>\lambda\right\} \leq C_{\max } \frac{\|f\|_{1}}{\lambda} .
$$

Proof of Proposition 7. By standard transference arguments, see for example [8] Chapter III, it is sufficient to establish a corresponding weak maximal inequality on the integers with $\lambda=1$ for nonnegative functions with compact support. Hence, this proof will be completed by Proposition 8 below.

Thus we need to state and prove the following maximal inequality:

Proposition 8. There exists a constant $C_{\max }$ such that for every $\varphi: \mathbb{Z} \rightarrow$ $[0, \infty)$ with compact support

$$
\#\left\{j: \sup _{K \in \mathbb{N}} M_{g, K} \varphi(j)>1\right\} \leq C_{\max }\|\varphi\|_{\ell_{1}} .
$$

Proposition 8 can also be reduced further to the following Claim. Set $M_{l}=M_{g, 2^{l}}$.

Claim 9. There exists a constant $C_{\max }^{\prime}$ such that for every $\varphi: \mathbb{Z} \rightarrow[0,+\infty)$ with compact support

$$
\#\left\{j: \sup _{l \in \mathbb{N}} M_{l} \varphi(j)>1\right\} \leq C_{\max }^{\prime}\|\varphi\|_{\ell_{1}}
$$


Proof of Proposition 8 based on Claim 9. Given $K \in \mathbb{N}$ choose $l_{K} \in \mathbb{N}$ such that $2^{l_{K}-1}<K \leq 2^{l_{K}}$. By (2), or (3) there exists a constant $C_{R}>0$ not depending on $K$ such that $S_{g, 2^{l} K} \leq C_{R} S_{g, K}$. We have

$$
\begin{gathered}
1<M_{g, K} \varphi(j)=\frac{1}{S_{g, K}} \sum_{j=1}^{K} g(n) \varphi(j+n) \leq \\
\frac{S_{g, 2^{l} K}}{S_{g, K}} \cdot \frac{1}{S_{g, 2^{l} K}} \sum_{n=1}^{2^{l} K} g(n) \varphi(j+n) \leq C_{R} M_{g, 2^{l} K} \varphi(j) .
\end{gathered}
$$

Hence, $1<M_{g, K} \varphi(j)$ implies $\frac{1}{C_{R}}<M_{g, 2^{l} K} \varphi(j)=M_{l_{K}} \varphi(j)$.

For any $\widetilde{\varphi}: \mathbb{Z} \rightarrow[0,+\infty)$ with compact support taking $\varphi=C_{R} \widetilde{\varphi}$ by Claim 9 we obtain

$$
\begin{gathered}
\#\left\{j: \sup _{K \in \mathbb{N}} M_{g, K} \widetilde{\varphi}(j)>1\right\} \leq \#\left\{j: \sup _{l \in \mathbb{N}} M_{l} \varphi(j)>1\right\} \leq \\
C_{\text {max }}^{\prime}\|\varphi\|_{\ell_{1}}=C_{\text {max }}^{\prime} C_{R}\|\widetilde{\varphi}\|_{\ell_{1}} .
\end{gathered}
$$

Proof of Claim 9. If $1 \leq l \leq 4$ then consider the set $E_{l}=\left\{j: M_{l} \varphi(j)>1\right\}$ and the system of intervals $\mathcal{I}_{l}=\left\{\left[j+1, j+2^{l}\right] \cap \mathbb{Z}: j \in E_{l}\right\}$. Then $E_{l}+1 \subset$ $\cup_{I \in \mathcal{I}_{l}} I$ and hence $\# E_{l} \leq \# \cup_{I \in \mathcal{I}_{l}} I$. We can select a subsystem $\mathcal{I}_{l}^{\prime} \subset \mathcal{I}_{l}$ such that no point of $\mathbb{Z}$ is covered by more than two intervals belonging to $\mathcal{I}_{l}^{\prime}$ and $\cup_{I \in \mathcal{I}_{l}^{\prime}} I=\cup_{I \in \mathcal{I}_{l}} I$.

Suppose $I=\left[j+1, j+2^{l}\right] \cap \mathbb{Z} \in \mathcal{I}_{l}^{\prime} \subset \mathcal{I}_{l}$. Then $M_{l} \varphi(j)>1$ implies that

$$
1<\frac{1}{S_{g, 2^{l}}} \sum_{n=1}^{2^{l}} g(n) \varphi(j+n),
$$

that is

$$
S_{g, 2^{l}} \leq \sum_{n=1}^{2^{l}} g(n) \varphi(j+n)=\sum_{k \in I} g(k-j) \varphi(k)
$$

Thus

$$
1 \leq \frac{S_{g, 2^{l}}}{\max _{k \leq 2^{l}} g(k)} \leq \sum_{k \in I} \varphi(k) .
$$


If $l \leq 4$ then we have $\# I / 16 \leq 1 \leq \sum_{k \in I} \varphi(k)$. Since no point is covered by more than two intervals $I \in \mathcal{I}_{l}^{\prime}$, that is, $\sum_{I \in \mathcal{I}_{l}^{\prime}} \chi_{I}(j) \leq 2,(j \in \mathbb{Z})$ we obtain that for $l \leq 4$

$$
\# E_{l} \leq \# \cup_{I \in \mathcal{I}_{l}^{\prime}} I \leq 32\|\varphi\|_{\ell_{1}}
$$

and hence

$$
\#\left\{j: \sup _{1 \leq l \leq 4} M_{l} \varphi(j)>1\right\} \leq 128\|\varphi\|_{\ell_{1}} .
$$

Next suppose that $l>4$. We consider the dyadic intervals $\left(r 2^{l},(r+1) 2^{l}\right] \cap$ $\mathbb{Z}, r \in \mathbb{Z}$. We say that $r \in R_{l,+}$ if

$$
\frac{1}{2^{l}} \sum_{j=r 2^{l}+1}^{r 2^{l}+2 \cdot 2^{l}} \varphi(j)>\frac{1}{100 \cdot C_{g, \max }} .
$$

Otherwise, if $r \notin R_{l,+}$ we say that $r \in R_{l,-}$.

For $r \in R_{l,-}$ we use Lemma 5 and the negation of (24) to deduce that for $l>4$

$$
\begin{gathered}
\sum_{j=1}^{2^{l}}\left(M_{l} \varphi\left(r 2^{l}+j\right)\right)^{\left\lfloor\log \log 2^{l}\right\rfloor}<\left(\sum_{j=2}^{2 \cdot 2^{l}} \varphi\left(r 2^{l}+j\right)\right) \cdot\left(\frac{1}{100}\right)^{\left\lfloor\log \log 2^{l}\right\rfloor-1} \leq \\
100^{2}\left(\sum_{j=2}^{2 \cdot 2^{l}} \varphi\left(r 2^{l}+j\right)\right) \cdot\left(\frac{1}{100}\right)^{\log \log 2^{l}} \leq \\
100^{2}\left(\sum_{j=2}^{2 \cdot 2^{l}} \varphi\left(r 2^{l}+j\right)\right) \cdot \exp \left(-(\log 100) \cdot \log \log 2^{l}\right) \leq \\
100^{2}\left(\sum_{j=2}^{2 \cdot 2^{l}} \varphi\left(r 2^{l}+j\right)\right) \cdot \frac{6}{l^{2}}, \text { where we used that }
\end{gathered}
$$

$4.61 \geq \log 100 \geq 4.60517$ and $\log \log 2>-0.37$ implies that

$$
\begin{gathered}
\exp \left(-(\log 100) \cdot \log \log 2^{l}\right)=\exp (-(\log 100)((\log l)+\log \log 2))= \\
\exp (-(\log 100) \log \log 2) \cdot \exp (-(\log 100) \log l)<\frac{6}{l^{2}} .
\end{gathered}
$$

Set $\mathcal{M}_{l}^{*}=\left\{j: M_{l} \varphi(j)>1\right\}$ and $\mathcal{M}^{*}=\cup_{l} \mathcal{M}_{l}^{*}$. 
If $r \in R_{l,-}$ then by (25)

$$
\begin{gathered}
\#\left(\mathcal{M}_{l}^{*} \cap\left(r 2^{l},(r+1) 2^{l}\right]\right) \leq \sum_{j=1}^{2^{l}}\left(M_{l} \varphi\left(r 2^{l}+j\right)\right)^{\left\lfloor\log \log 2^{l}\right\rfloor} \leq \\
6 \cdot 100^{2} \cdot \frac{1}{l^{2}}\left(\sum_{j=2}^{2 \cdot 2^{l}} \varphi\left(r 2^{l}+j\right)\right) .
\end{gathered}
$$

Hence

$$
\begin{gathered}
\#\left(\mathcal{M}_{l}^{*} \cap \bigcup_{r \in R_{l,-}}\left(r 2^{l},(r+1) 2^{l}\right]\right) \leq \\
12 \cdot 100^{2} \frac{1}{l^{2}}\left\|\varphi_{\ell_{1}}\right\|
\end{gathered}
$$

and

$$
\#\left(\bigcup_{l}\left(\mathcal{M}_{l}^{*} \cap \cup_{r \in R_{l,-}}\left(r 2^{l},(r+1) 2^{l}\right]\right)\right) \leq 12 \cdot 100^{2} \frac{\pi^{2}}{6}\left\|\varphi_{\ell_{1}}\right\| .
$$

On the other hand,

$$
\cup_{l>4} \cup_{r \in R_{l,+}}\left(r 2^{l},(r+1) 2^{l}\right] \cap \mathbb{Z} \subset \cup_{l>4} \cup_{r \in R_{l,+}}\left[r 2^{l},(r+2) 2^{l}\right] \cap \mathbb{Z} .
$$

We can again select a subsystem $\mathcal{I}_{+}^{*}$ of the intervals $\mathcal{I}_{+}=\left\{\left[r 2^{l},(r+2) 2^{l}\right]\right.$ : $\left.l>4, r \in R_{l,+}\right\}$ such that

$$
\sum_{I \in \mathcal{I}_{+}^{*}} \chi_{I}(j) \leq 2 \text { for all } j \in \mathbb{Z} \text { and } \cup_{I \in \mathcal{I}_{+}} I=\cup_{I \in \mathcal{I}_{+}^{*}} I .
$$

From (24) it follows that if $\left[r 2^{l},(r+2) 2^{l}\right]=I \in \mathcal{I}_{+}^{*}$ then

$$
C_{g, \max } \cdot 400 \sum_{j \in I} \varphi(j)>4 \cdot 2^{l}>\#(I \cap \mathbb{Z}) .
$$

Thus, by (28)

$$
\#\left(\cup_{I \in \mathcal{I}_{+}} I \cap \mathbb{Z}\right)=\#\left(\cup_{I \in \mathcal{I}_{+}^{*}} I \cap \mathbb{Z}\right)<C_{g, \max } \cdot 800\|\varphi\|_{\ell_{1}} .
$$

Hence, by (27)

$$
\#\left(\cup_{l>4} \cup_{r \in R_{l,+}}\left(r 2^{l},(r+1) 2^{l}\right] \cap \mathbb{Z}\right) \leq C_{g, \max } \cdot 800\|\varphi\|_{\ell_{1}} .
$$

From this, (23) and (26) it follows that

$$
\# \mathcal{M}^{*} \leq\left(128+12 \cdot 100^{2} \frac{\pi^{2}}{6}+800 C_{g, \max }\right)\|\varphi\|_{\ell_{1}}=C_{\max }^{\prime}\|\varphi\|_{\ell_{1}} .
$$




\section{References}

[1] C. Cuny and M. Weber, Ergodic theorems with arithmetical weights, Preprint:https://arxiv.org/abs/1412.7640.

[2] H. Delange, Sur des formules de Atle Selberg, Acta Arith. 19, 105-146, (1971).

[3] E. H. El Abdalaoui, J. Kułaga-Przymus, M. Lemańczyk and T. De La Rue, The Chowla and the Sarnak conjectures from ergodic theory point of view, Preprint:https://arxiv.org/abs/1410.1673.

[4] G. Halász, On the distribution of additive and the mean values of multiplicative arithmetic functions, Studia Sci. Math. Hungar., 6, 211-233, (1971).

[5] G.H. Hardy and E. M. Wright, An introduction to the theory of numbers. Sixth edition. Oxford University Press, Oxford, (2008).

[6] K. K. Norton, On the number of restricted prime factors of an integer. I. Illinois J. Math. 20, no. 4, 681-705, (1976).

[7] K. Petersen, Ergodic Theory, Cambridge Studies in Advanced Mathematics 2, Cambridge University Press, (1981).

[8] J. M. Rosenblatt and M. Wierdl, Pointwise ergodic theorems via harmonic analysis. Ergodic theory and its connections with harmonic analysis (Alexandria, 1993), 3-151, London Math. Soc. Lecture Note Ser., 205, Cambridge Univ. Press, Cambridge, (1995). 